\title{
An Improved Method to Calculate the Conservation of Mass in the Simulation of Rapid Pressurization and Depressurization in a Packed Bed
}

\author{
Thomas S.Y. Choong \\ Corresponding author. Department of Chemical \\ and Environmental Engineering, Universiti Putra Malaysia, \\ 43400 Serdang, Malaysia. Fax: (603)- 86567099; E-mail: tsyc2@eng.upm.edu.my \\ William R. Paterson and David M. Scott \\ Department of Chemical Engineering, University of \\ Cambridge, Pembroke Street, Cambridge, CB2 3RA, U.K.
}

The work described here forms part of a project to model rapid pressure swing adsorption (RPSA), which is a single-bed process used for air separation. We have earlier identified a form of model and boundary conditions for an axially dispersed plug flow model that conserves mass. We solve the RPSA models numerically by spatially discretizing the partial differential equations to a system of ordinary differential equations (ODEs), which are then integrated over time. Although the formulation of our models conserves mass, our numerical simulations, however, do not perfectly conserve mass because of discretization error and rounding error. The discrepancy in the conservation of mass is computed as a guide to the numerical accuracy of the calculations. The computation of the conservation error requires the evaluation of time integrals of molar flowrates in and out of the bed. Since the velocity at the feed end of the bed changes rapidly with time, the application of quadrature to evaluate the time integrals does not provide the accuracy required. In this paper, the inadequacy is demonstrated using a simple problem, i.e. pressurization and depressurization into a non-adsorptive bed. An improved method is proposed. By transforming equations involving time integrals into ODEs, excellent accuracy is obtained. Further, this transformation minimizes the number of decision parameters that need to be specified by the users of the computer programs.

Keywords: rapid pressure swing adsorption, modelling and simulation, packed bed.

\section{INTRODUCTION}

We aim to model rapid pressure swing adsorption (RPSA), which is a single-bed process used for air separation. It operates with very short cycle times (in the order of seconds) and uses small adsorbent particles (typically $200-700 \mathrm{~mm}$ in diameter). A basic RPSA cycle consists of two steps: pressurization and depressurization, as illustrated in Figure 1. During pressurization, air is fed to the column through a three-way valve. Pressure increases rapidly at the feed end of the column. As feed air flows down the column, nitrogen is preferentially adsorbed on the zeolite $5 \mathrm{~A}$ adsorbent, resulting in an oxygen-enriched gas phase. In the depressurization step, the feed valve is closed and the exhaust valve at the feed end is opened to atmospheric pressure, resulting in a 


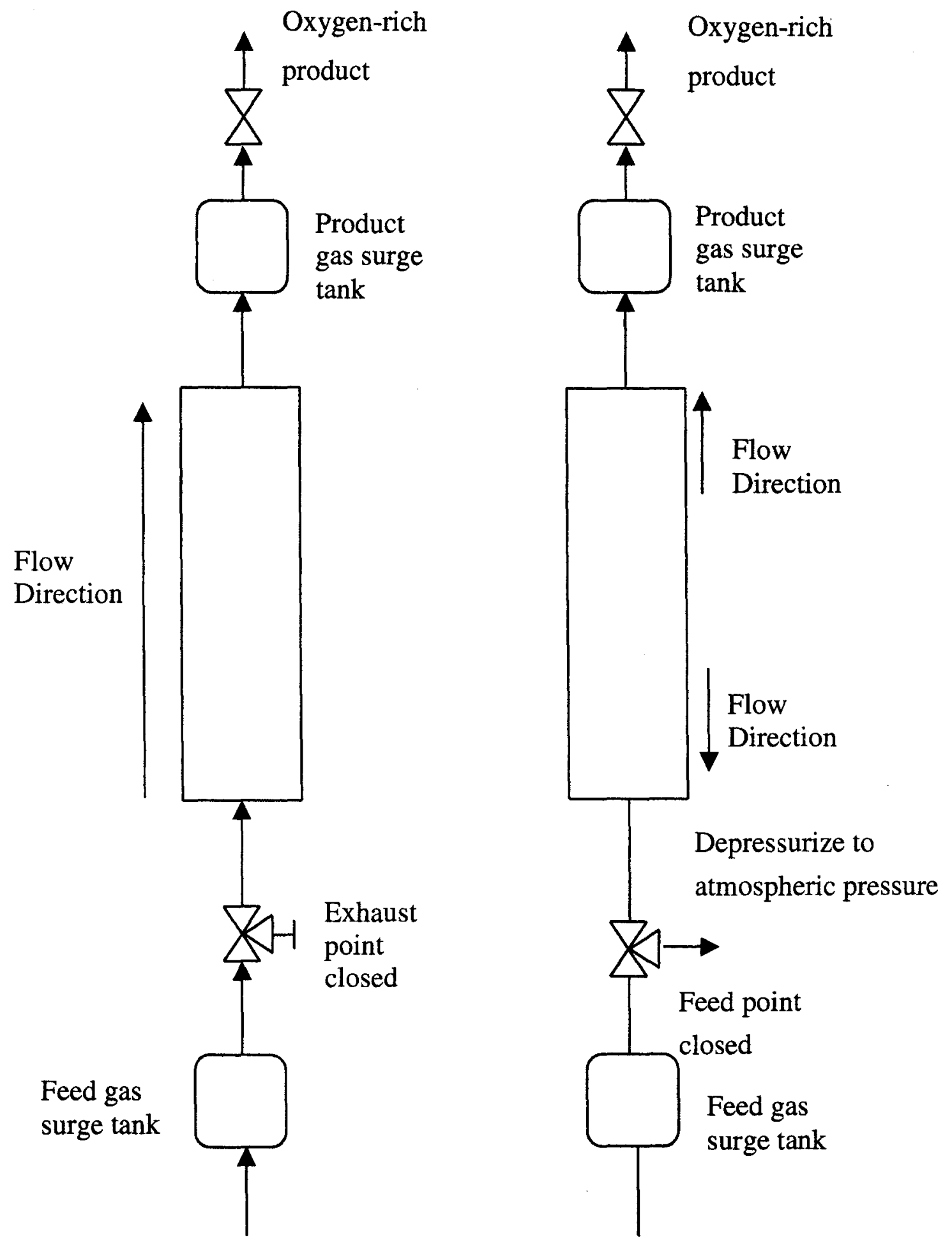

Pressurize with air

(a) Pressurization

(b) Depressurization

Figure 1: Basic steps in RPSA 
rapid pressure drop at the feed end of the column, followed by desorption of the adsorbed nitrogen. The gas leaving the exhaust port is enriched with nitrogen. As there is a pressure maximum in the bed during depressurization, a pressure gradient is always maintained between this maximum and the product end of the bed, which results in a continuous product stream throughout the cycle. In RPSA, flow resistance in the bed is crucial for successful operation of the process.

We have earlier identified a form of model and boundary conditions for an axially dispersed plug flow model that conserves mass (Choong et al., 1998). The mass-conserved RPSA models are solved numerically by spatially discretizating the partial differential equations (PDEs) to a system of ordinary differential equations (ODEs), which are then integrated over time. Although the formulation of our models conserves mass, our numerical simulations, however, do not perfectly conserve mass because of discretization error and rounding error. We compute the discrepancy in the conservation of mass as a guide to the numerical accuracy of the calculations. The computation of the conservation error requires the evaluation of time integrals of molar flowrates in and out of the bed. The time integrals are often evaluated using quadrature (Alpay et al., 1993). However, as the velocity at the feed end of the bed changes rapidly with time, the application of quadrature to evaluate the time integrals does not provide the accuracy required. In this paper, we demonstrate this inadequacy using a simple problem, i.e. pressurization and depressurization into a non-adsorptive bed. An improved method is then proposed. By transforming equations involving time integrals into ODEs, excellent accuracy is obtained. Further, this transformation minimizes the number of decision parameters that need to be specified by the users of the computer programs.

\section{A simple problem: integration for a non-adsorptive bed}

Consider pressurization and depressurization of air in a non-adsorptive bed packed with nonporous solid with the product end closed. The process is approximated as isothermal and the flow pattern as plug flow. The Ideal Gas law is assumed to apply. A step change of pressure is assumed at the feed end of the bed. The total material balance yields

$$
\frac{\partial P}{\partial t}=\frac{-1}{\varepsilon_{b}}\left(u \frac{\partial P}{\partial z}+P \frac{\partial u}{\partial z}\right)
$$

where $P$ is pressure $\left(\mathrm{N} / \mathrm{m}^{2}\right), t$ is time $(\mathrm{s}), z$ is an axial co-ordinate $(\mathrm{m}), u$ is the local superficial gas velocity $\left(\mathrm{m} \mathrm{s}^{-1}\right)$ and $\varepsilon_{b}$ is the bed porosity. The gas flow in the packed bed is described by Darcy's law

$$
\frac{\partial P}{\partial z}=-J_{v} u=-180 \frac{\mu\left(1-\varepsilon_{b}\right)^{2}}{d_{p}^{2} \varepsilon_{b}^{3}} u
$$

where $J_{v}$ is commonly known as the bed permeability $\left(\mathrm{N} \mathrm{s} \mathrm{m}^{4}\right), \mu$ is the gas viscosity $\left(\mathrm{N} \mathrm{s} \mathrm{m}^{-2}\right)$ and $d_{p}$ is the particle diameter $(\mathrm{m})$. Rearranging equation (2) gives the local superficial velocity

$$
u=\frac{-1}{J_{v}} \frac{\partial P}{\partial z}
$$

\section{Initial conditions}

Pressurization

$$
\text { For all z: } P_{\text {init }}=P_{\text {am }}
$$

Depressurization

$$
\text { For all z: } P_{\text {init }}=5 P_{\text {am }}
$$

where the subscripts $a m$ and init refer to ambient conditions $\left(P_{\mathrm{am}}=1 \times 10^{5} \mathrm{~N} \mathrm{~m}^{-2}\right)$ and initial conditions, respectively.

\section{Boundary conditions}

\section{Pressurization}

$z=0: P=5 P_{\mathrm{am}}$ and $z=L: \quad \partial P / \partial z=0$

Depressurization

$z=0: \quad P=P_{\text {am }}$ and $z=L: \quad \partial P / \partial z=0$ 
where $L$ is the length of the bed $(m)$. Because of discretization error and rounding error, our numerical simulations will not perfectly conserve mass. The conservation error is computed as a guide to the numerical accuracy of our calculations. At any time during a pressurization step, the cumulative molar amount fed to the bed, $M_{\text {feed }}$, must, in principle, equal the sum of the cumulative molar amount accumulated in the bed, $M_{\text {accum. }}$ In a depressurization step, $M_{\text {feed }}$ will be the cumulative molar amount leaving the bed from the feed end. However, due to discretization error and rounding error, the equality will never be exact. The total material balance relative error is calculated from:

$$
E r r=\left|\frac{M_{\text {feed }}-M_{\text {accum }}}{M_{\text {feed }}}\right| \times 100 \%
$$

Table 1a Pressurization: Effect of order of polynomials (J) on the accuracy of quadrature for evaluating the changes of gas phase in the bed.

\begin{tabular}{|c|c|c|c|c|}
\hline \multirow{2}{*}{$t^{\prime}(\mathrm{s})$} & \multicolumn{4}{|c|}{$\bar{M}_{\text {accum }}$} \\
\cline { 2 - 5 } & $J=10$ & $J=20$ & $J=30$ & $J=50$ \\
\hline 1 & $1.2392(0.065)$ & $1.2386(0.016)$ & $1.2385(0.008)$ & 1.2384 \\
2 & $1.5262(0.013)$ & $1.5261(0.007)$ & $1.5260(0.000)$ & 1.5260 \\
3 & $1.5863(0.000)$ & $1.5863(0.000)$ & $1.5863(0.000)$ & 1.5863 \\
4 & $1.5975(0.000)$ & $1.5975(0.000)$ & $1.5975(0.000)$ & 1.5975 \\
5 & $1.5995(0.006)$ & $1.5996(0.000)$ & $1.5995(0.006)$ & 1.5996 \\
10 & $1.6000(0.000)$ & $1.6000(0.000)$ & $1.6000(0.000)$ & 1.6000 \\
20 & $1.6000(0.000)$ & $1.6000(0.000)$ & $1.6000(0.000)$ & 1.6000 \\
30 & $1.6000(0.000)$ & $1.6000(0.000)$ & $1.6000(0.000)$ & 1.6000 \\
40 & $1.6000(0.000)$ & $1.6000(0.000)$ & $1.6000(0.000)$ & 1.6000 \\
\hline
\end{tabular}

Table $1 \mathrm{~b}$ Depressurization: Effect of order of polynomials $(J)$ on the accuracy of quadrature for evaluating the changes of gas phase in the bed.

\begin{tabular}{|c|c|c|c|c|}
\hline \multirow{2}{*}{$t^{\prime}(\mathrm{s})$} & \multicolumn{4}{|c|}{$\bar{M}_{\text {accum }}$} \\
\cline { 2 - 5 } & $J=10$ & $J=20$ & $J=30$ & $J=50$ \\
\hline 1 & $-0.9978(0.160)$ & $-0.9992(0.020)$ & $-0.9993(0.010)$ & -0.9994 \\
2 & $-1.2665(0.060)$ & $-1.2671(0.008)$ & $-1.2672(0.000)$ & -1.2672 \\
3 & $-1.3959(0.021)$ & $-1.3962(0.000)$ & $-1.3962(0.000)$ & -1.3962 \\
4 & $-1.4682(0.010)$ & $-1.4684(0.000)$ & $-1.4684(0.000)$ & -1.4684 \\
5 & $-1.5121(0.007)$ & $-1.5122(0.000)$ & $-1.5122(0.000)$ & -1.5122 \\
10 & $-1.5858(0.006)$ & $-1.5859(0.000)$ & $-1.5859(0.000)$ & -1.5859 \\
20 & $-1.5995(0.000)$ & $-1.5995(0.000)$ & $-1.5995(0.000)$ & -1.5995 \\
30 & $-1.6000(0.000)$ & $-1.6000(0.000)$ & $-1.6000(0.000)$ & -1.6000 \\
40 & $-1.6000(0.000)$ & $-1.6000(0.000)$ & $-1.6000(0.000)$ & -1.6000 \\
\hline
\end{tabular}

( ) the number in brackets denotes the relative \% difference between simulation results and simulation results for $J=50$, defined as $\%$ difference $=\left|\frac{\text { simulation }- \text { simulation }(J=50)}{\text { simulation }(J=50)}\right| \times 100 \%$ 
where

$$
M_{\text {feed }}=A \int_{0}^{t^{\prime}}(u c)_{f} d t=\frac{A \int_{0}^{t^{\prime}}(u P)_{f} d t}{R_{g} T}
$$

and

$M_{\text {accum }}=\varepsilon_{b} A \int_{0}^{L}\left(c-c_{\text {init }}\right) d z=\frac{\varepsilon_{b} A \int_{0}^{L}\left(P-P_{\text {init }}\right) d z}{R_{g} T}$

where $R_{g}$ is the Ideal Gas constant $\left(8.314 \mathrm{~J} \mathrm{~mol}^{-1}\right.$ $\left.\mathrm{K}^{-1}\right), T$ is temperature (K), $A$ is the bed cross sectional area $\left(\mathrm{m}^{2}\right)$ and $t^{\prime}$ is any time measured from the start of a step. The subscript $f$ refers to positions far upstream of $z=0$.

The process parameters used in the simulations are: particle diameter, $d_{p}=100 \mathrm{~mm}$ bed porosity, $\varepsilon_{b}=0.4$; bed diameter, $d_{c}=0.05 \mathrm{~m}$; temperature, $T=290 \mathrm{~K}$; bed length, $L=1.0 \mathrm{~m}$; viscosity of air, $\mathrm{m}=1.81 \cdot 10^{-5} \mathrm{~N} \mathrm{~s} \mathrm{~m}^{-2}$. The total time for each step considered is $40 \mathrm{~s}$.

\section{Numerical method}

The PDE of equation (1) is discretized in the space domain to a system of ODEs using the orthogonal collocation (OC) method, which are then integrated over time. More information on

Table 2a Pressurization: Effect of quadrature points in the evaluation of time integrals of molar flowrates at the feed end of the bed. The order of polynomial used is 20. IT is the number of equally spaced quadrature points.

\begin{tabular}{|c|c|c|c|c|c|c|c|c|c|}
\hline \multirow{2}{*}{$t^{\prime}(\mathrm{s})$} & \multicolumn{3}{|c|}{$I T=50$} & \multicolumn{3}{c|}{$I T=1000$} & \multicolumn{3}{c|}{$I T=10000$} \\
\cline { 2 - 10 } & $\bar{M}_{\text {feed }}$ & $\bar{M}_{\text {accum }}$ & $\operatorname{Err}(\%)$ & $\bar{M}_{\text {feed }}$ & $\bar{M}_{\text {accum }}$ & $\operatorname{Err}(\%)$ & $\bar{M}_{\text {feed }}$ & $\bar{M}_{\text {accum }}$ & $\operatorname{Err}(\%)$ \\
\hline 1 & 1.1262 & 1.2386 & 9.99 & 1.2116 & 1.2386 & 2.23 & 1.2278 & 1.2386 & 0.88 \\
2 & 1.3676 & 1.5261 & 11.59 & 1.4902 & 1.5261 & 2.41 & 1.5134 & 1.5261 & 0.84 \\
3 & 1.3924 & 1.5863 & 13.93 & 1.5423 & 1.5863 & 2.85 & 1.5721 & 1.5863 & 0.90 \\
4 & 1.3738 & 1.5975 & 16.28 & 1.5462 & 1.5975 & 3.32 & 1.5818 & 1.5975 & 0.99 \\
5 & 1.3496 & 1.5996 & 18.52 & 1.5425 & 1.5996 & 3.70 & 1.5820 & 1.5996 & 1.11 \\
10 & 1.2470 & 1.6000 & 28.31 & 1.5201 & 1.6000 & 5.26 & 1.5731 & 1.6000 & 1.71 \\
20 & 1.0999 & 1.6000 & 45.47 & 1.4876 & 1.6000 & 7.56 & 1.5642 & 1.6000 & 2.29 \\
30 & 0.9973 & 1.6000 & 60.43 & 1.4627 & 1.6000 & 9.39 & 1.5561 & 1.6000 & 2.82 \\
40 & 0.9155 & 1.6000 & 74.77 & 1.4416 & 1.6000 & 10.99 & 1.5487 & 1.6000 & 3.31 \\
\hline
\end{tabular}

Table 2b Depressurization: Effect of quadrature points in the evaluation of time integrals of molar flowrates at the feed end of the bed. The order of polynomial used is 20. IT is the number of equally spaced quadrature points.

\begin{tabular}{|c|c|c|c|c|c|c|c|c|c|}
\hline \multirow{2}{*}{$t^{\prime}(\mathrm{s})$} & \multicolumn{3}{|c|}{$I T=50$} & \multicolumn{3}{c|}{$I T=1000$} & \multicolumn{3}{c|}{$I T=10000$} \\
\cline { 2 - 9 } & $\bar{M}_{\text {feed }}$ & $\bar{M}_{\text {accum }}$ & Err $(\%)$ & $\bar{M}_{\text {feed }}$ & $\bar{M}_{\text {accum }}$ & $\operatorname{Err}(\%)$ & $\bar{M}_{\text {feed }}$ & $\overline{\bar{M}}_{\text {accum }}$ & Err $(\%)$ \\
\hline 1 & -0.9086 & -0.9992 & 9.97 & -0.9830 & -0.9992 & 1.65 & -0.9958 & -0.9992 & 0.34 \\
2 & -1.1378 & -1.2671 & 11.36 & -1.2417 & -1.2671 & 2.05 & -1.2619 & -1.2671 & 0.41 \\
3 & -1.2372 & -1.3962 & 12.85 & -1.3638 & -1.3962 & 2.38 & -1.3893 & -1.3962 & 0.50 \\
4 & -1.2845 & -1.4684 & 14.32 & -1.4302 & -1.4684 & 2.67 & -1.4600 & -1.4684 & 0.58 \\
5 & -1.3064 & -1.5122 & 15.75 & -1.4690 & -1.5122 & 2.94 & -1.5023 & -1.5122 & 0.66 \\
10 & -1.2941 & -1.5859 & 22.55 & -1.5229 & -1.5859 & 4.14 & -1.5696 & -1.5859 & 1.04 \\
20 & -1.1881 & -1.5995 & 34.63 & -1.5089 & -1.5995 & 6.00 & -1.5740 & -1.5995 & 1.62 \\
30 & -1.0932 & -1.6000 & 46.36 & -1.4883 & -1.6000 & 7.51 & -1.5675 & -1.6000 & 2.07 \\
40 & -1.0080 & -1.6000 & 58.73 & -1.4706 & -1.6000 & 8.80 & -1.5618 & -1.6000 & 2.45 \\
\hline
\end{tabular}


Table 3a Pressurization: Accuracy achieved after the transformation of time integral to $O D E$. The order of polynomial used is 20.

\begin{tabular}{|c|c|c|c|}
\hline$t^{\prime}(\mathrm{s})$ & $\bar{M}_{\text {feed }}$ & $\bar{M}_{\text {accum }}$ & Err (\%) \\
\hline 1 & 1.2390 & 1.2386 & 0.0347 \\
2 & 1.5265 & 1.5261 & 0.0288 \\
3 & 1.5867 & 1.5863 & 0.0277 \\
4 & 1.5980 & 1.5975 & 0.0325 \\
5 & 1.6000 & 1.5995 & 0.0288 \\
10 & 1.6005 & 1.6000 & 0.0312 \\
20 & 1.6005 & 1.6000 & 0.0312 \\
30 & 1.6005 & 1.6000 & 0.0312 \\
40 & 1.6005 & 1.6000 & 0.0312 \\
\hline
\end{tabular}

Table 3b Depressurization: Accuracy achieved after the transformation of time integral to ODE. The order of polynomial used is 20.

\begin{tabular}{|c|c|c|c|}
\hline$t^{\prime}(\mathrm{s})$ & $\bar{M}_{\text {feed }}$ & $\bar{M}_{\text {accum }}$ & Err (\%) \\
\hline 1 & -0.9985 & -0.9992 & -0.0691 \\
2 & -1.2664 & -1.2671 & -0.0584 \\
3 & -1.3955 & -1.3962 & -0.0494 \\
4 & -1.4676 & -1.4684 & -0.0518 \\
5 & -1.5115 & -1.5122 & -0.0470 \\
10 & -1.5851 & -1.5859 & -0.0479 \\
20 & -1.5988 & -1.5996 & -0.0469 \\
30 & -1.5993 & -1.6000 & -0.0431 \\
40 & -1.5993 & -1.6000 & -0.0438 \\
\hline
\end{tabular}

the method of OC can be found in Rice and Do (1995). The computer program is written in FORTRAN 77 for the implementation of modelling and simulation. The program coding and simulation are carried out on a SUN ULTRA Enterprise $2(170 \mathrm{MHz})$ workstation. Standard algorithms from the NAG FORTRAN library (Mark 16) are employed as external subroutines. The ODE integration algorithm employed the NAG FORTRAN library subroutine D02EJF, which is based on a variable order, variable step method implementing a backward differentiation formulae, and is suitable for a stiff system of first order non-linear ODEs. The accuracy of the integration is controlled by the absolute tolerance, TOL, used in the subroutine. The value of TOL $=1^{\prime} 10^{-5}$ is used in all simulations. The NAG subroutine D01GAF, which employs a third order finite difference method, is used for quadrature.

\section{QUADRATURE}

The quadrature of the space integral in equation (10) is first considered. The number of points in the quadrature is equal to the number of collocation points in the space domain. Therefore, no additional adjustable parameter is introduced. We consider polynomials of five different orders. The simulation results are reported in Tables $1 \mathrm{a}$ and $1 \mathrm{~b}$ for pressurization and depressurization, respectively. For both cases, a polynomial of order 20 is considered sufficient for the evaluation of the space integral. Therefore, subsequent simulations using the $\mathrm{OC}$ method are 
carried out with a polynomial of order 20 .

To evaluate the time integral in equation (9), quadrature using NAG subroutine D01GAF with equally spaced time points is employed. The accuracy of the quadrature depends on the number of time points used in the NAG subroutine D01GAF. As shown in Tables 2a and 2b, 10,000 equally spaced time points are required for the quadrature using NAG subroutine D01GAF to maintain a total material balance relative error of about $1 \%$ for short pressurization and depressurization times $(<5 \mathrm{~s})$. For pressurization and depressurization times larger than 5 seconds, however, even use of 10,000 time points failed to reduce the total material balance relative error to $1 \%$. This clearly demonstrates the inadequacy of quadrature for the evaluation of time integrals when velocity changes rapidly with time at the feed end of the bed. The accuracy of the quadrature may be further improved by using a larger number of time points or by concentrating the time points in the region of the steepest velocity profile (Shampine et al., 1997). However, the former approach may increase the computing time substantially, and since the velocity profile at the feed end of the bed is usually unknown a priori, the latter approach may not be easily implemented.

\section{A USEFUL TRANSFORMATION}

An alternative approach is now used. Differentiate equation (9) with respect to $t$ to find

$$
\frac{d M_{\text {feed }}}{d t}=\frac{A}{R_{g} T}(u P)_{f}
$$

The initial condition for equation (11) is:

$$
T=0, \quad M_{\text {feed }}=0 .
$$

Equations (11) and (12) can now be added to the set of ODEs and initial conditions arising from discretizing the PDE. The accuracy of the evaluation of the time integral is now maintained by the ODE solver.
As shown in Tables $3 a$ and $3 b$, by transforming equation (9) into an ODE, a total material balance relative error of $<0.1 \%$ can be easily achieved for all pressurization and depressurization times using a polynomial of order 20. This clearly demonstrates the superiority of the simple transformation in overcoming the steep velocity variation at the feed end of the bed with time. Another advantage obtained from the transformation is that no additional decision parameter is introduced for the evaluation of the time integral. With the transformation, the accuracy of the time intregral is maintained automatically by the ODE solver. Provided a suitable ODE solver is employed, good accuracy can always be obtained in the evaluation of the time integral. With the transformation, the accuracy of the numerical calculations depends only on the spatial discretization and the tolerance used in the ODE integration algorithm. The complete algorithm for the modelling and simulation of RPSA will be reported later.

\section{CONCLUSION}

The error in conservation of mass is computed as a guide to the numerical accuracy of the calculations. The computation of the conservation error requires the evaluation of time integrals of molar flowrates into and out of the bed. Since the velocity at the feed end of the bed changes rapidly with time, the application of quadrature does not provide the accuracy required. This difficulty is overcome by transforming all the equations involving time integrals into ODEs. The superiority of the transformation over quadrature is demonstrated by considering a simple problem of pressurization and depressurization of air into a non-adsorptive packed bed. This transformation also minimizes the number of decision parameters that need to be specified for the computer program. With the transformation, the accuracy of the numerical calculations depends only on the spatial discretization and the tolerance used in the ODE integration algorithm. 


\section{NOTATION}

A bed cross sectional area $\left(\mathrm{m}^{2}\right)$

c total gas phase concentration $\left(\mathrm{mol} \mathrm{m}^{-3}\right)$

Err relative material balance error (\%)

$d_{p} \quad$ particle diameter $(m)$

$d_{c} \quad$ column diameter $(m)$

$\mathrm{J}$ order of polynomial in the method of OC

$\mathrm{J}_{\mathrm{v}} \quad$ bed permeability $\left(\mathrm{N} \mathrm{s} \mathrm{m}^{-4}\right)$

$\mathrm{L} \quad$ length of bed $(\mathrm{m})$

$\mathrm{M} \quad$ cumulative molar amount at any time during a step

$\bar{M}=\frac{R_{g} T}{A P_{a n}} M$ dimensionless cumulative molar amount

$\mathrm{P} \quad$ total bed pressure $(\mathrm{Pa})$

$\mathrm{R}_{\mathrm{g}} \quad$ ideal gas constant $\left(\mathrm{J} \mathrm{mol}^{-1} \mathrm{~K}^{-1}\right)$

$\mathrm{u} \quad$ superficial gas velocity $\left(\mathrm{m} \mathrm{s}^{-1}\right)$

$\mathrm{t}$ time (s)

$\mathrm{t}^{\prime} \quad$ any time measured from the start of a step (s)

$\mathrm{T}$ temperature $(\mathrm{K})$

$z \quad$ axial co-ordinate $(\mathrm{m})$

\section{Greek letters}

$\varepsilon_{\mathrm{b}} \quad$ bed porosity

$\mathrm{m} \quad$ gas viscosity $\left(\mathrm{N} \mathrm{s} \mathrm{m}^{-2}\right)$

\section{Subscripts}

$\begin{array}{ll}\text { am } & \text { ambient conditions } \\ \mathrm{f} & \text { accumulation in the bed } \\ \text { feed } & \text { feed end of the bed } \\ \text { init } & \text { initial values }\end{array}$

\section{ACKNOWLEDGEMENTS}

T.S.Y.C. would like to thank the Overseas Development Administration (ODA) and Universiti Putra Malaysia (UPM) for financial support.

\section{REFERENCES}

Alpay, E., Kenney, C. N. and Scott, D. M., "Simulation of Rapid Pressure Swing Adsorption and Reaction Processes", Chem. Engng Sci. 1993, 48, pp 3173 3186.

Choong, T. S. Y., Paterson, W. R. and Scott, D. M., "Axial Ddispersion in Rich, Binary Gas Mixtures: Model Form and Boundary Conditions", Chem. Engng Sci., 1998, 53, pp 4147 - 4149.

Rice, R. G. and Do, D. D., Applied Mathematics and Modelling for Chemical Engineers, John Wiley \& Sons, U.S.A. (1995).

Shampire, L. F., Allen, R.C. and Pruess, S., Fundamentals of Numerical Computing, John Wiley \& Sons, New York (1997). 